Supplement of Solid Earth, 6, 9-31, 2015

http://www.solid-earth.net/6/9/2015/

doi:10.5194/se-6-9-2015-supplement

(C) Author(s) 2015. CC Attribution 3.0 License.

(c) (i)

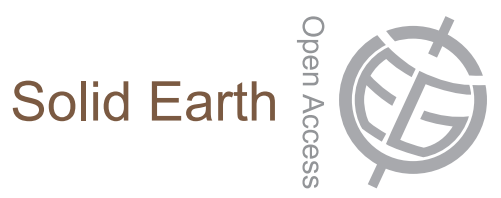

Supplement of

\title{
Analogue experiments of salt flow and pillow growth due to basement faulting and differential loading
}

M. Warsitzka et al.

Correspondence to: M. Warsitzka (michael.warsitzka@uni-jena.de) 


\section{A Extension rate e}
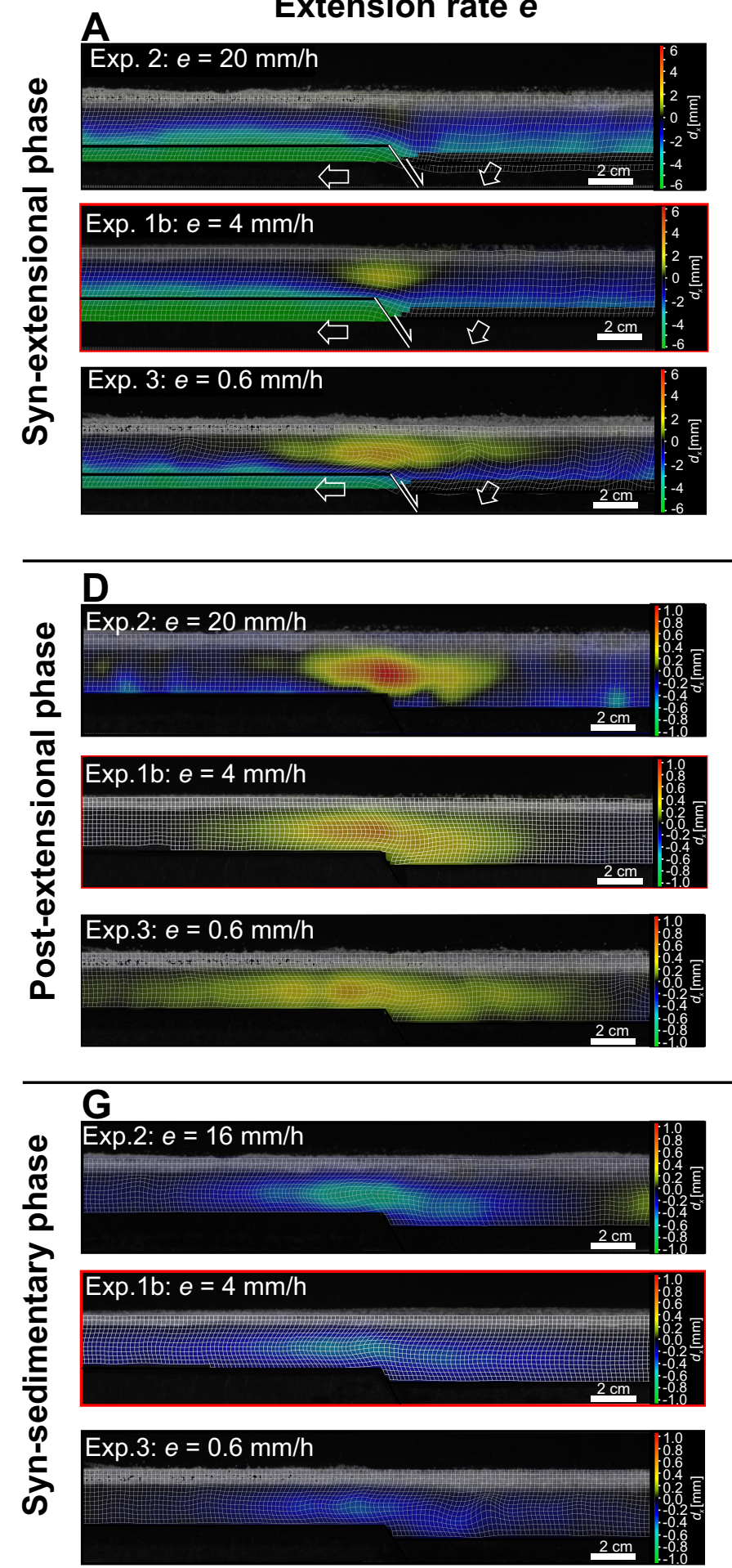

Figure C1. Parameter study of horizontal displacement $d$ above the tip of the basement fault during the syn-extensional, view). Coloured areas display rightward (yellow-red) and leftward (green-blue) movement of the analogue mate
basement $e(\mathbf{A}, \mathbf{D}, \mathbf{G})$, thicknesses of the viscous layer $h_{d}(\mathbf{B}, \mathbf{E}, \mathbf{H})$, and thicknesses of the cover layer $h_{b}(\mathbf{C}, \mathbf{F}, \mathbf{I})$.
C Thickness cover layer $h_{b}$

Exp. 4: $h_{d}=10 \mathrm{~mm}$

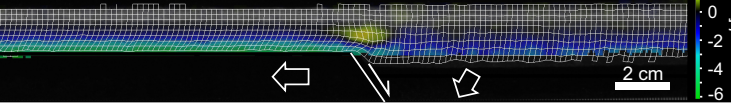
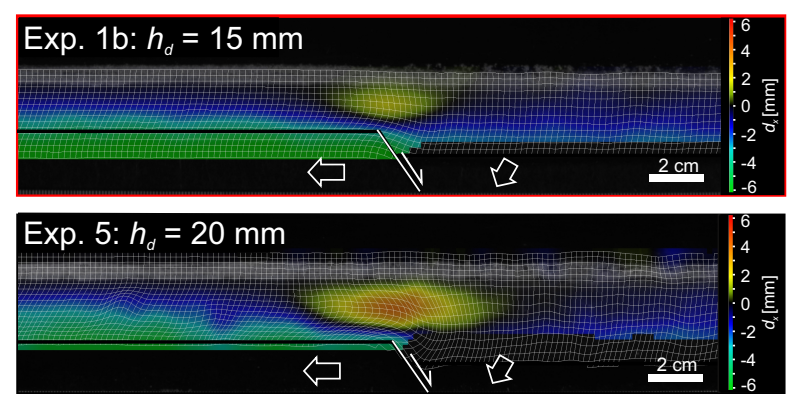

E
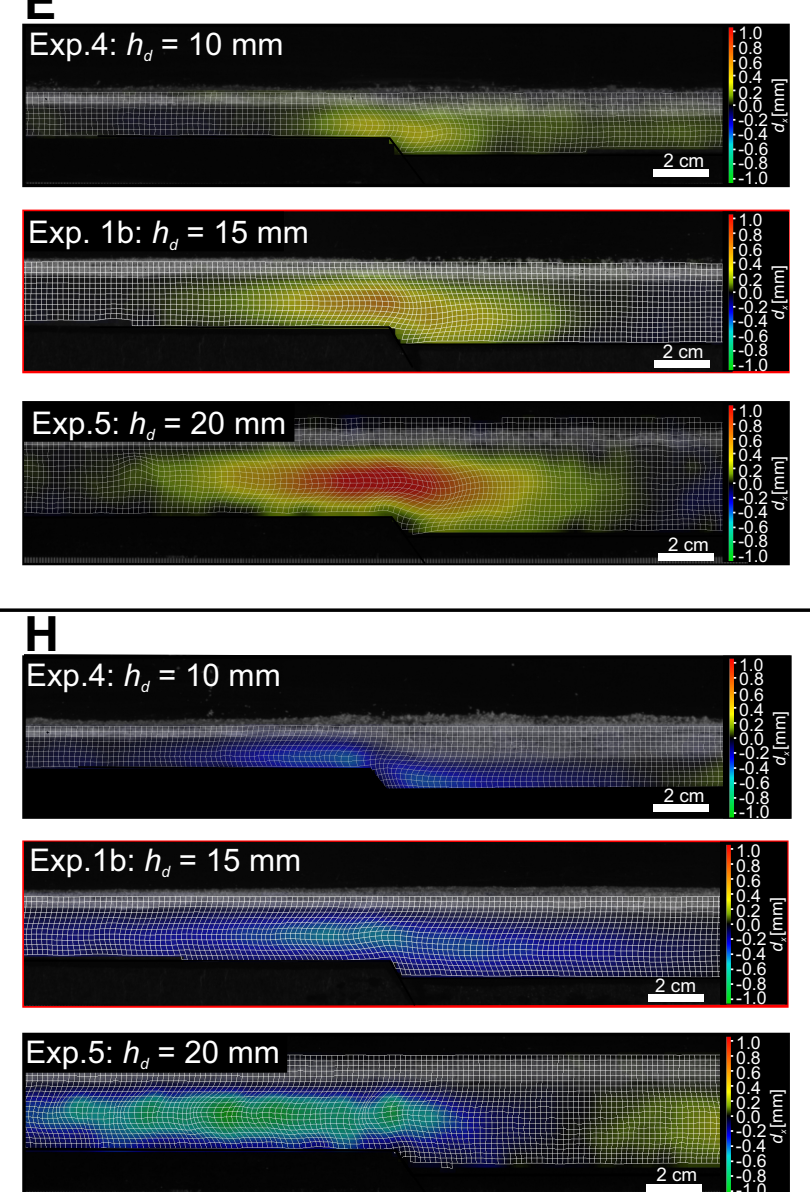
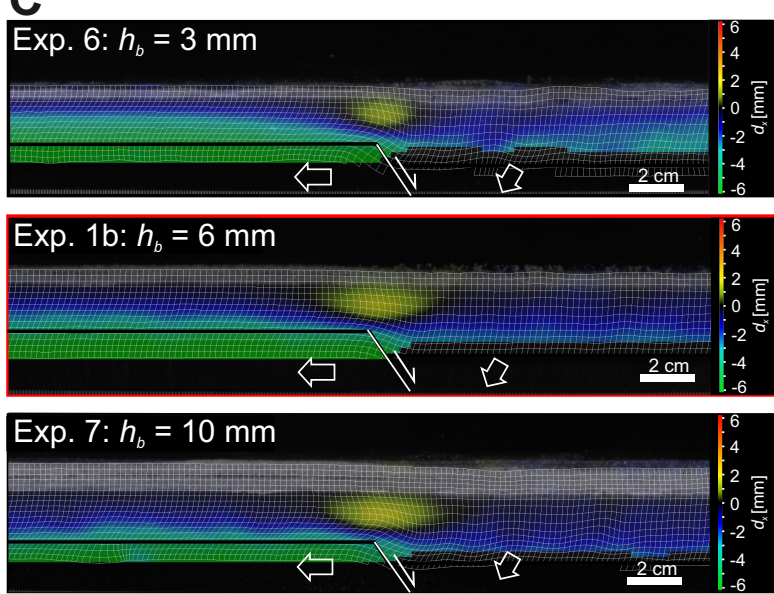

$\mathbf{F}$

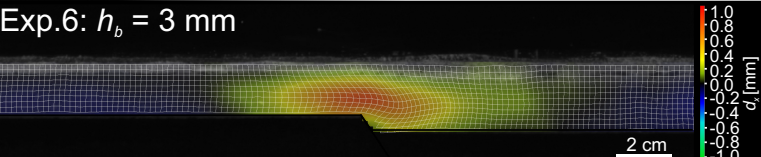

Exp. $1 \mathrm{~b}: h_{b}=6 \mathrm{~mm}$

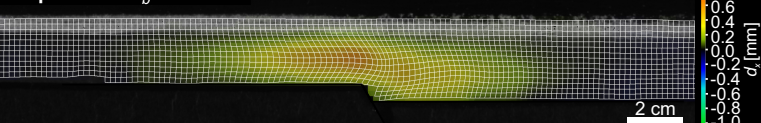

Exp.7: $h_{b}=10 \mathrm{~mm}$

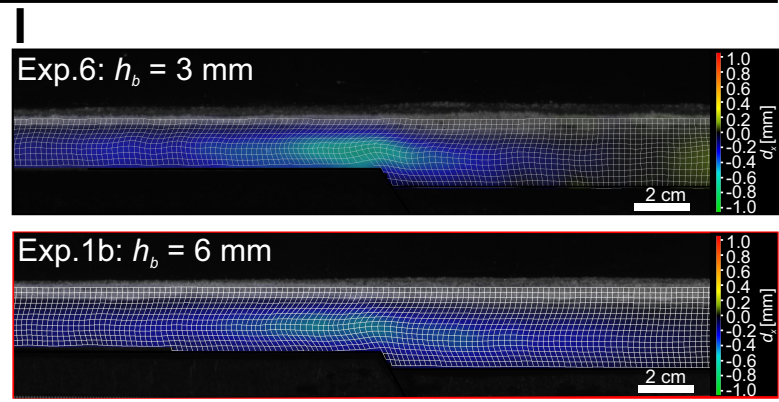

\section{Exp.7: $h_{b}=10 \mathrm{~mm}$}

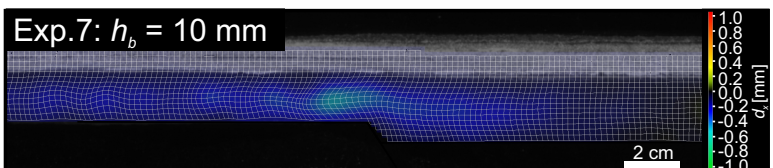

Exp.6: $h_{b}=3 \mathrm{~mm}$ 


\section{A Dependence of structures on the extension rate e}

Exp. 2: $h_{d}=15 \mathrm{~mm} ; h_{b}=6 \mathrm{~mm} ; e=0.6 \mathrm{~mm} / \mathrm{h}$
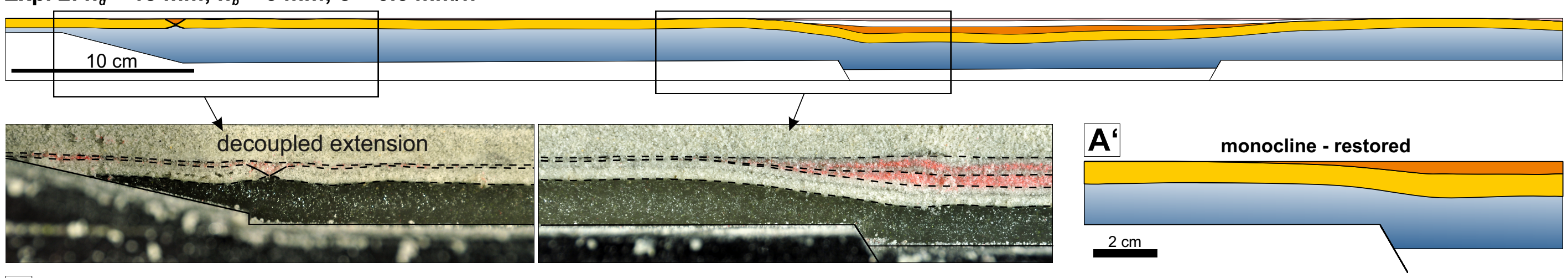

B

Exp. 1c: $h_{d}=15 \mathrm{~mm} ; h_{b}=6 \mathrm{~mm}$; e $=4 \mathrm{~mm} / \mathrm{h}$
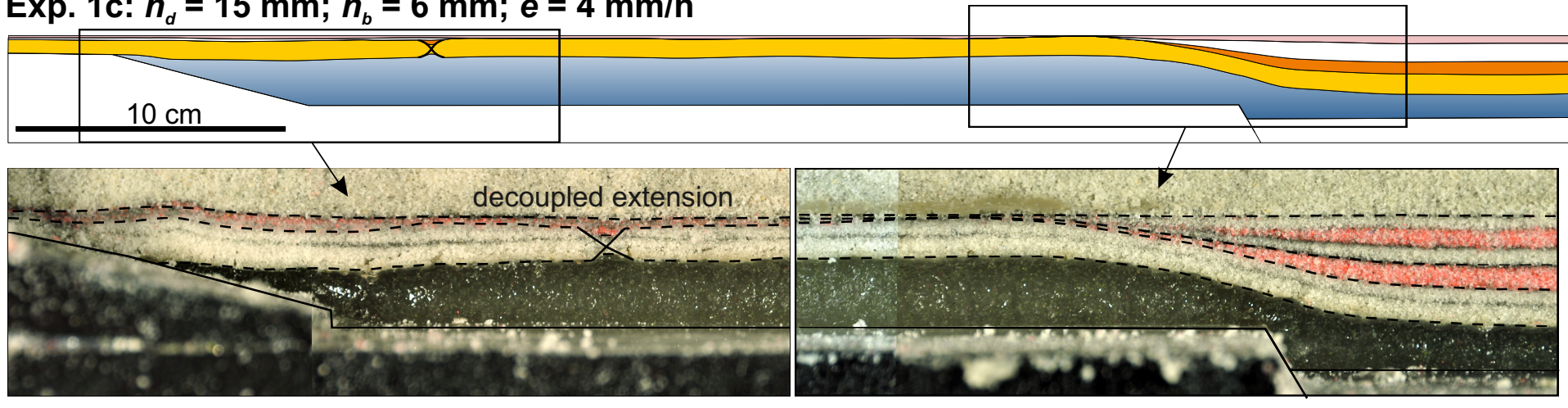

B monocline - restored

C

Exp. 3: $h_{d}=15 \mathrm{~mm} ; h_{b}=6 \mathrm{~mm} ; e=20 \mathrm{~mm} / \mathrm{h}$
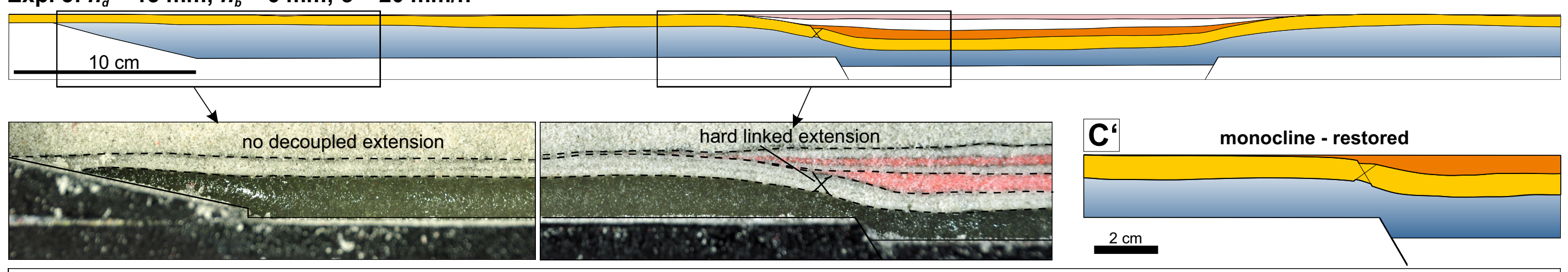

Legend:

$\square$ Silicone

$\square$ Pre-extensional sand layer

1st sand layer added

$\Longleftarrow$ Post-extensional sand layers

Figure C2. Comparison of experimental structures (after 4 days) depending on the basement extension rate $e$. In experiments with slow basement extension (A/B), the cover graben is located in larger distance from the basement graben compared to experiments with fast basement extension (C). With increasing basement extension rate, the monocline in the cover layer becomes narrower and the subsidence of the hanging wall peripheral sink increases $\left(\mathbf{A}^{\prime} / \mathbf{B}^{\prime} / \mathbf{C}^{\prime}\right)$. 
Exp. 4a: $h_{d}=10 \mathrm{~mm} ; h_{b}=6 \mathrm{~mm} ; e=4 \mathrm{~mm} / \mathrm{h}$
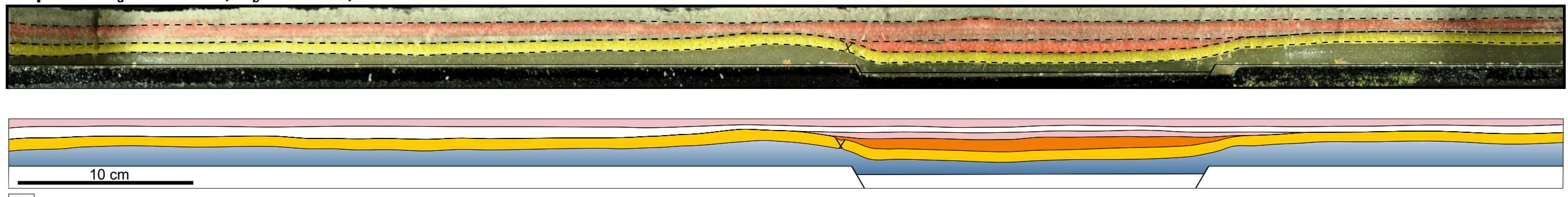

B

Exp. 1a: $h_{d}=15 \mathrm{~mm} ; h_{b}=6 \mathrm{~mm} ; e=4 \mathrm{~mm} / \mathrm{h}$
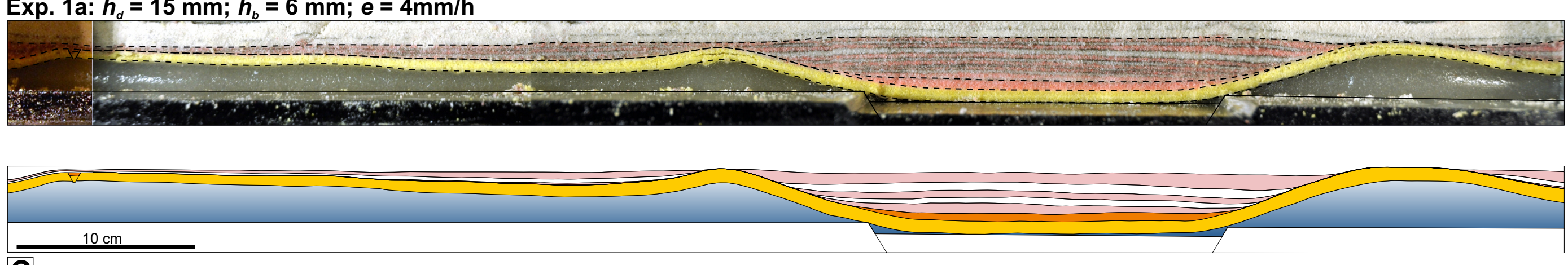

C

Exp. 5: $h_{d}=20 \mathrm{~mm} ; h_{b}=6 \mathrm{~mm} ; e=4 \mathrm{~mm} / \mathrm{h}$
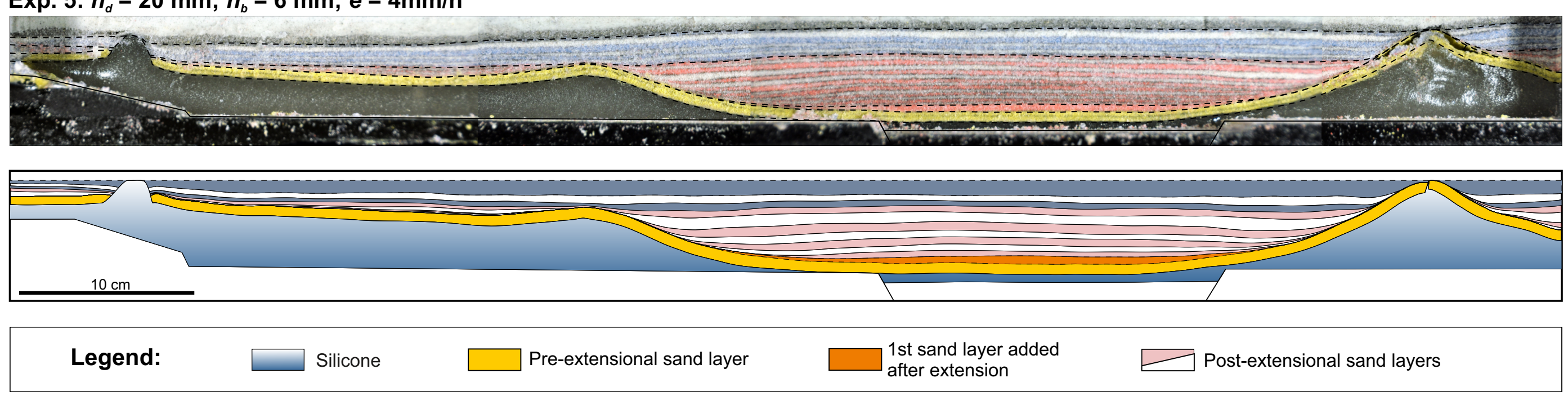

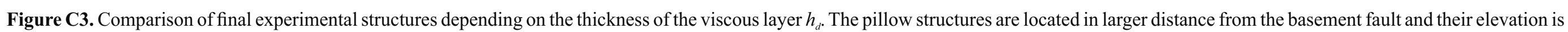
higher when increasing $h_{b}$. In the experiment with thickest $h_{b}(\mathbf{C})$, the viscous material pierced the overburden layer. 


\section{$\mathbf{A}$}

Dependence of structures on the thickness of the cover layer $h_{b}$

Exp. 6: $h_{d}=15 \mathrm{~mm} ; h_{b}=3 \mathrm{~mm} ; e=4 \mathrm{~mm} / \mathrm{h}$

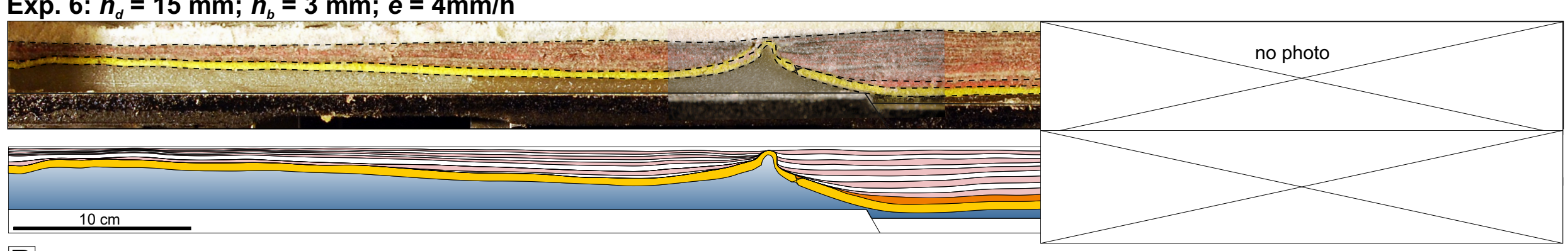

B

Exp. 1a: $h_{d}=15 \mathrm{~mm} ; h_{b}=6 \mathrm{~mm} ; e=4 \mathrm{~mm} / \mathrm{h}$

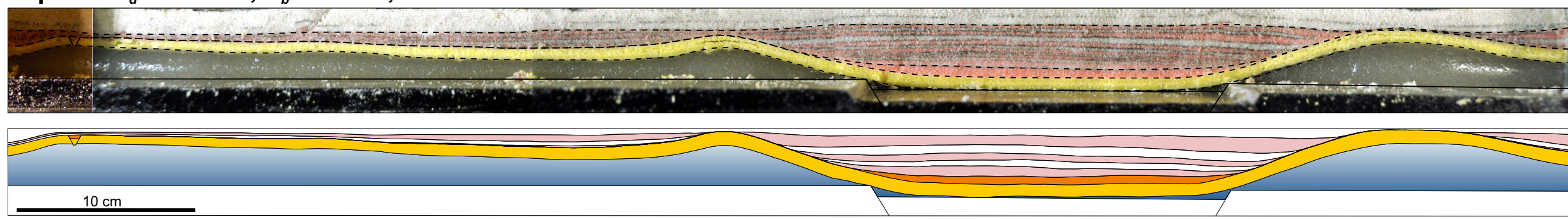

C

Exp. 7: $h_{d}=15 \mathrm{~mm} ; h_{b}=10 \mathrm{~mm} ; e=4 \mathrm{~mm} / \mathrm{h}$

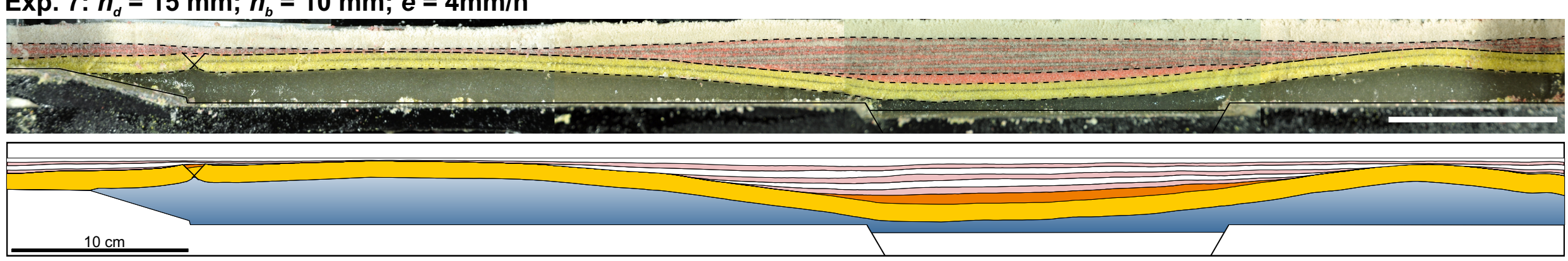

D

Exp. 8: $h_{d}=15 \mathrm{~mm} ; h_{b}=15 \mathrm{~mm} ; e=4 \mathrm{~mm} / \mathrm{h}$

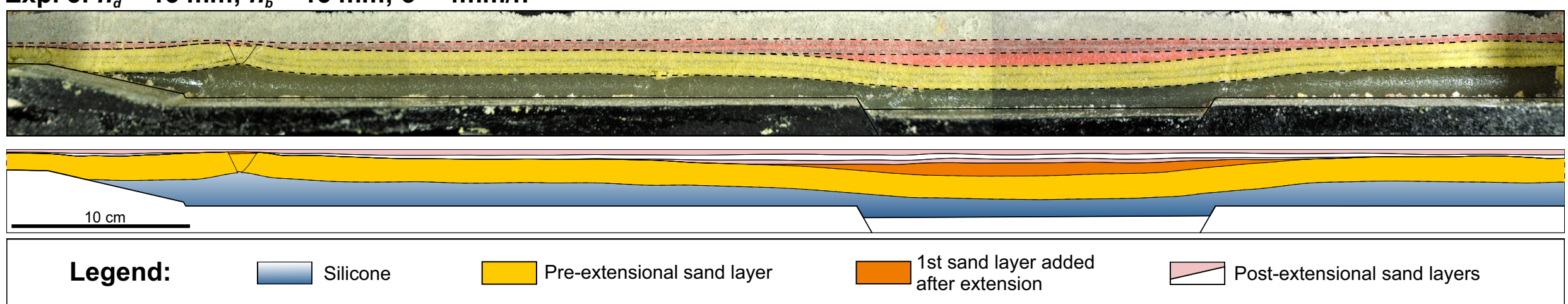

Figure C4. Parameter study of final experimental structures depending on the thickness of the pre-kinematic cover layer $h_{b}$. The width of the pillow structures is wider and their elevation is smaller when increasing $h_{b}$. In the experiment with the thickest pre-kinematic cover layer (Exp. $8 ;(\mathbf{C})$ ), no pronounced pillow structures evolved. 
A

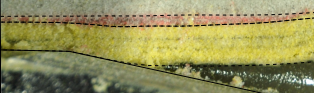

$10 \mathrm{~cm}$

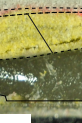

-1 -

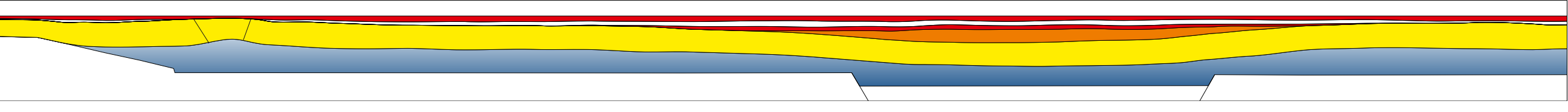

B Syn-extensional phase

$\frac{10 \mathrm{~cm}}{\text { Post-extensional phase }}$

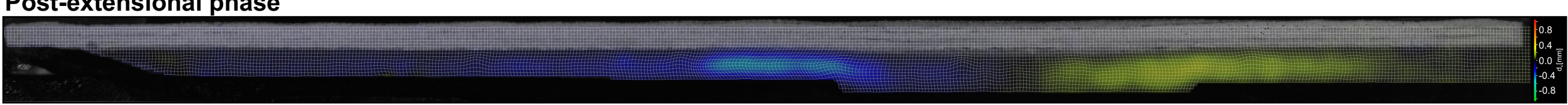

Syn-sedimentary phase (after 5 days)

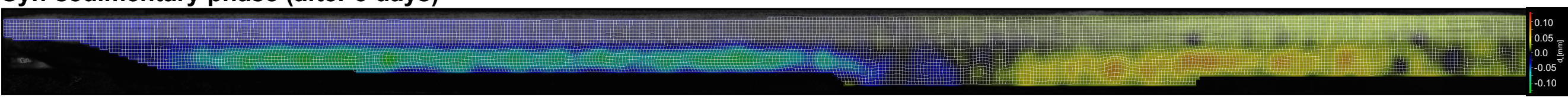

\begin{tabular}{|c|c|c|c|c|}
\hline Legend: & $\square$ Silicone & $\begin{array}{l}\text { 1st sand layer added } \\
\text { after 1st phase of extension }\end{array}$ & Pre-extensional sand layer & $\begin{array}{l}\text { Post-extensional sand layers } \\
\text { after 1st phase of extension }\end{array}$ \\
\hline
\end{tabular}

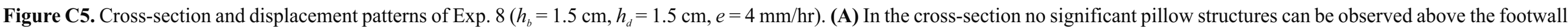

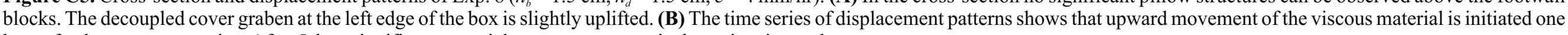
hour after basement extension. After 5 days significant material movement occurs in the entire viscous layer. 
Tab. C1: Data for plot in Figure 11

Dependence on basement extension rate e

Experimental stage Displacement rate in the viscous layer above the basement fault tip $[\mathrm{mm} / \mathrm{h}]$

$\operatorname{Exp} \cdot 2(e=20 \mathrm{~mm} / \mathrm{h}) \quad \operatorname{Exp} \cdot 1 \mathrm{~b}(e=4.0 \mathrm{~mm} / \mathrm{h}) \operatorname{Exp} \cdot 3(e=0.6$

$\mathrm{mm} / \mathrm{h})$

$\begin{array}{llll}\text { syn-extensional } & & 1.8 & 1.43 \\ \text { post-extensional } & 4.73 & 2.65 & 1.08 \\ \text { syn-sedimentary } & 10.77 & 0.50 & 0.40 \\ \text { syn-sedimentary } & 20.87 & 0.60 & 0.48 \\ \text { syn-sedimentary } & 30.99 & 0.56 & 0.56 \\ \text { syn-sedimentary } & 41.0 & 0.60 & 0.54\end{array}$

Dependence on thickness of the viscous layer hd

Experimental stage Displacement rate in the viscous layer above the basement fault tip $[\mathrm{mm} / \mathrm{h}]$

Exp. $4($ hd $=1 \mathrm{~cm}) \quad \operatorname{Exp} .1(\mathrm{hd}=1.5 \mathrm{~cm}) \quad \operatorname{Exp} .5(\mathrm{hd}=2$

$\mathrm{cm}$ )

syn-extensional $\quad 0.60 \quad 1.43 \quad 2.5$

post-extensional $2.11 \quad 2.65 \quad 4.97$

$\begin{array}{llll}\text { syn-sedimentary } & 10.31 & 0.50 & 0.74\end{array}$

$\begin{array}{llll}\text { syn-sedimentary } & 20.35 & 0.60 & 0.80\end{array}$

syn-sedimentary $30.52 \quad 0.56 \quad 0.70$

$\begin{array}{llll}\text { syn-sedimentary } & 40.55 & 0.60 & 0.75\end{array}$

Dependence on thickness of the cover layer hb

Experimental stage Displacement rate in the viscous layer above the basement fault tip $[\mathrm{mm} / \mathrm{h}]$

$\operatorname{Exp} .6(\mathrm{hb}=0.3 \mathrm{~cm}) \quad \operatorname{Exp} \cdot 1(\mathrm{hd}=1.5 \mathrm{~cm}) \quad \operatorname{Exp} \cdot 7(\mathrm{hb}=1$ $\mathrm{cm}$ )

$\begin{array}{lllll}\text { syn-extensional } & & 1.00 & 1.43 & 1.43 \\ \text { post-extensional } & 4.00 & 2.65 & 2.08 & \\ \text { syn-sedimentary } & 10.70 & 0.50 & 0.36 & \\ \text { syn-sedimentary } & 20.75 & 0.60 & 0.46 & \\ \text { syn-sedimentary } & 31.05 & 0.56 & 0.46 \\ \text { syn-sedimentary } & 40.80 & 0.60 & 0.5\end{array}$

